\title{
Plasma mass filtering for separation of actinides from lanthanides
}

\author{
R Gueroult and N J Fisch \\ Princeton Plasma Physics Laboratory, Princeton University, Princeton, NJ 08543, USA \\ E-mail: rgueroul@pppl.gov
}

Received 13 January 2014, revised 4 March 2014

Accepted for publication 11 March 2014

Published 16 April 2014

\begin{abstract}
Separating lanthanides from actinides is a key process in reprocessing nuclear spent fuel. Plasma mass filters, which operate on dissociated elements, offer conceptual advantages for such a task as compared with conventional chemical methods. The capabilities of a specific plasma mass filter concept, called the magnetic centrifugal mass filter, are analyzed within this particular context. Numerical simulations indicate separation of americium ions from a mixture of lanthanides ions for plasma densities of the order of $10^{12} \mathrm{~cm}^{-3}$, and ion temperatures of about $10 \mathrm{eV}$. In light of collision considerations, separating small fractions of heavy elements from a larger volume of lighter ones is shown to enhance the separation capabilities.
\end{abstract}

Keywords: plasma filter, confinement, numerical simulation

(Some figures may appear in colour only in the online journal)

\section{Introduction}

The utility of plasmas to discriminate elements based on their mass has long been recognized. It can be traced as far back as the calutron device $[1,2]$ during the Manhattan project. The potential of rotating plasma configurations was quickly identified [3]. These configurations present the advantage, as compared with their classic gaseous or liquid counterparts, to operate at larger rotation speeds, offering in principle faster processing rates.

The vast majority of the research conducted in this field has been dedicated to isotope separation [4-6], for which the mass difference of the elements to be separated is small. This small mass difference in turn usually limits the achievable throughput.

More recently, plasma mass filtering has been considered for nuclear waste remediation [7]. In this case, the mass difference can be significant, allowing theoretically much larger throughputs. Two different configurations relying on different mass discrimination schemes have been proposed to fulfill this objective. In both cases, the separation device is at essence a plasma source or confinement device or, put more precisely, a differential plasma confinement, device, or plasma filter.

In the Ohkawa filter [8], mass separation is achieved as a result of the existence of a charge-to-mass threshold for ion confinement. Unconfined heavy ions are therefore collected radially, while light elements exit axially. On the other hand, in the magnetic centrifugal mass filter (MCMF) $[9,10]$, ions of different masses are collected at different axial ends of the device. This is made possible using asymmetric collisional rotating plasma.

The fact that plasma mass filters operate irrespectively of the input stream chemical form makes them attractive for separation processes, which are simultaneously chemically challenging and of high added value. Among these is the separation of lanthanides from actinides, within the process of nuclear spent fuel reprocessing [11]. Partitioning and transmutation of the long-lived minor actinides into shorterlived or stable elements is considered as a way to decrease the mandatory storage time of remaining nuclear waste to a few hundred years, in place of several thousand years $[12,13]$. However, a prerequisite to this step consists in the separation of lanthanides from minor actinides because of the large lanthanides' neutron capture cross section [14]. From a chemical standpoint, this separation is made particularly difficult by the chemical similarities existing between the two groups of elements $[11,15]$.

In this paper, the potential of the MCMF is evaluated with respect to separating lanthanides from actinides. First, in section 2, the MCMF operating principles are recalled, and the production of a plasma out of an input stream is 


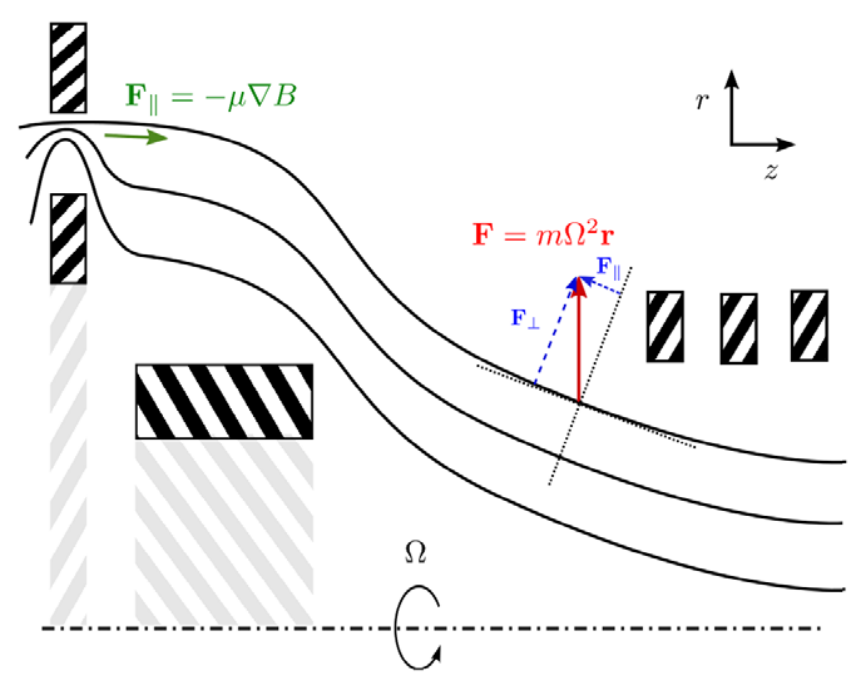

Figure 1. Cut view of the MCMF magnetic field topology (the $r-z$ plane). Solid lines represent the magnetic field lines; the dashed-dotted line indicates the axis of symmetry; hatched boxes represent magnetic coils.

described. In section 3, a numerical modeling of test ion behavior within the plasma is conducted to illustrate the dependence of plasma confinement properties on ion mass. These simulations allow estimating realistic plasma parameters for the considered separation process. In section 4, the model is then refined to analyze the influence of a multi-species plasma on the separation properties. These results highlight the asymmetric role played by the plasma composition on the plasma confinement properties, that is to say on separation. In section 5 , the main findings are summarized.

\section{Magnetic centrifugal mass filter}

The particular plasma mass filter considered here is the socalled MCMF [9], which consists of an axisymmetric rotating plasma. As illustrated in figure 1, mass separation in this device relies on asymmetric confinement properties at each end of the device. Plasma confinement at one end is dominated by centrifugal forces, and is thus mass dependent. At the other end, plasma confinement is dominated by magnetic forces, which are mass independent. The requirements of mass discrimination with respect to plasma parameters have been previously discussed [10]. Among these is a collisionality sufficiently large to ensure the ion diffusion by means of ionion pitch-angle scattering.

In the MCMF concept, the plasma production and operation will rely on a combination of radio-frequency (RF) and dc fields. RF antennas surrounding the plasma will be used to heat electrons through helicon waves [16], while a set of magnet coils will provide the static magnetic field topology depicted in figure 1. In addition, sets of concentric electrodes positioned at each axial ends of the device will be used to bias the plasma and establish a dc electric field perpendicularly to the static magnetic field lines. This electric field will in turn provide both ion heating and plasma rotation through the $\boldsymbol{E} \times \boldsymbol{B}$ drift. The main source of electron heating is through wave-particle interactions; the main source of ion heating is acceleration in the dc electric field. Electrons are too light to absorb much power through rotation, while ions are too massive to absorb much RF power.

Although a solid body rotation profile can ideally be produced through a proper electrode design [7], collisionality, as well as magnetic field non-uniformity, might lead to sheared rotation profiles. The rotation profile exact form will depend on multiple parameters, notably the plasma density and the electron temperature. Since our goal here is to identify relevant plasma parameters for a particular separation process, the exact rotation profile is undetermined at this point. As a consequence, and although a sheared rotation profile might alter the separation estimates, a solid body rotation profile will be assumed in the rest of this paper. It is, however, worth noting that sheared rotation profiles might be used to produce radial separation [19], in addition to the axial separation offered by a solid body rotating MCMF.

Various schemes for introducing and ionizing material into the plasma can be considered. One option would consist in injecting the material to be filtered in the form of a powder, allowing subsequent evaporation and ionization in the plasma. Although the powder grain size to be injected will depend on the plasma parameters [17], micrometer-size powder is likely to be required to ensure ionization of the input stream. In addition to the various chemical elements present in the input stream and, as a result, in the plasma, a buffer gas could be injected to provide additional control over the plasma parameters. As an example, the addition of a buffer gas would offer an additional indirect control knob on the electron temperature, which is thought to have strong effects on line radiation losses [18].

\section{Evaluation of the plasma filter performances}

A high added value separation process for which a plasma filter appears attractive consists in the separation of actinides from lanthanides, as encountered in the study of nuclear fuel cycle optimization. As described in the appendix, the transmutation of minor actinides presupposes separating lanthanides from the spent fuel stream. In particular, one ideally wants to isolate $\mathrm{Am}^{241}$ from a mixture of lanthanides. From a plasma mass filtering perspective, this could be idealized as processing a plasma made of a mixture of two elements: americium, with a mass of $241 \mathrm{amu}$, and a $144 \mathrm{amu}$ ion representative of lanthanide elements. This simplified plasma composition will be studied in the rest of this paper.

\subsection{Numerical model}

Numerical simulations can be used to assess the potential of the plasma filter for a particular separation process, that is to say a particular plasma composition, as well as to identify the relevant plasma parameters (number densities, temperatures, rotation speed). The physics of the numerical model used here is described elsewhere [10]. Here, we only give a brief summary of the model. It consists in a 3D modeling of individual ion trajectories within the rotating 


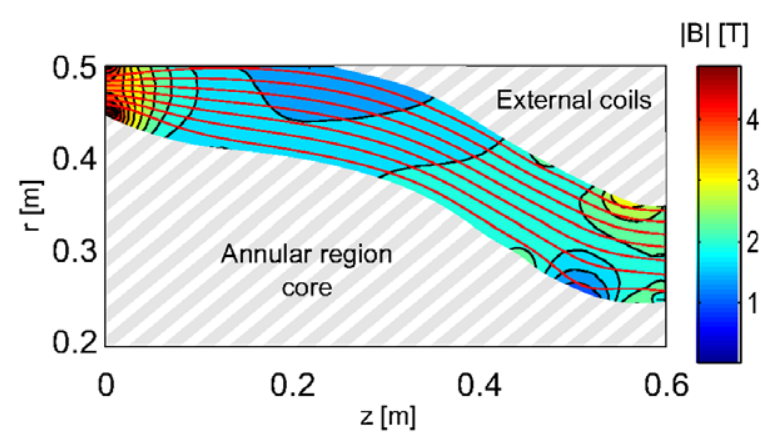

Figure 2. Magnetic field map used for the simulations. Red lines represent the magnetic field lines; hatched regions depict, respectively, the inner core region, used to bend the magnetic field lines, and the outer region, where the magnetic coils producing the magnetic field are positioned. The region of interest is therefore annular.

collisional plasma. Ion motion is calculated from the Lorentz force resulting from the externally prescribed electric and magnetic fields, as well as from Coulomb collisions computed in the rotating frame. Collisions with the background rotating plasma are computed in a Langevin formalism [20]. Selfgenerated fields are neglected. The simulation is run until the particle exits through one of the axial ends of the device, or, alternatively, is lost radially. Separation properties are estimated by computing statistics over a large number of ion trajectories.

The device dimensions considered here are identical to the ones previously used [10], namely $50 \mathrm{~cm}$ in radius and $60 \mathrm{~cm}$ in length. However, the magnetic and electric field strength and topology differ slightly. The magnetic field map is plotted in figure 2. As compared with [10], the magnetic field topology has been modified to maximize the radial extent of the confinement at the mirror throat (heavy element extraction side). The electric field, set by specifying a given electric potential to individual magnetic field line, dictates the rotation speed through the $\boldsymbol{E} \times \boldsymbol{B}$ drift.

\subsection{Relevant plasma parameters and typical achievable separation}

A first step toward assessing the MCMF separation capabilities consists in simulating the behavior of a test ion in a plasma made of its parent species and electrons, e.g. a $\mathrm{Am}^{241}$ ion interacting with a plasma made of $\mathrm{Am}^{241}$ ions and electrons. The results obtained for an ion of $144 \mathrm{amu}$ as well as for a ${ }^{241} \mathrm{Am}$ ion are given in figure 3 . The results globally display a relatively large spectrum of separation values while maintaining the radial losses under $15 \%$. As an example, for a plasma number density of $10^{12} \mathrm{~cm}^{-3}$ and a rotation speed $v_{\boldsymbol{E} \times \boldsymbol{B}}$ at the ion injection point of about $4.25 \mathrm{~km} \mathrm{~s}^{-1}$ (data points in gray in figure 3), about three ${ }^{241} \mathrm{Am}$ ions are lost to the heavy side for one lost to the light side. For the same parameters, this ratio is about 1.33 for the 144 amu test ion. Looking at the rotation speed dependence, one can see that an increase in the rotation speed enhances the mass dependence of the properties, which is depicted by an increase in the distance between same color data points. This trend is combined with a decrease in the collection at the light side, in favor of the heavy side, as a result of the larger centrifugal potential barrier to be overcome at the light side end. This translates into a shift of the data points to the left in figure 3. Moving now to the influence of the plasma density, an increase in $n$ leads to a combined increase in the collection on the light side (shift to the right) and of the radial losses (shift upward). The light side collection increase can be explained by the higher collisionality, which enhances the ion-ion pitch angle scattering allowing ion extraction on the light side [10]. The higher collisionality also explains the radial loss increase, since the ion motion perpendicular to the magnetic field lines results from collisional diffusion.

These preliminary simulation results indicate that the MCMF is suitable to operate on lanthanides and americium, for plasma densities up to a few $10^{12} \mathrm{~cm}^{-3}$. However, these results have been obtained by simulating the interaction of a single ion with a plasma made of its sole parent species and electrons. Since the MCMF separation scheme relies on ionion collisions, it stands to reason that a modification of the plasma composition would affect the separation properties of this device. In addition, as discussed in the appendix, the plasma composition expected for the particular americiumlanthanide separation process is asymmetric, with lanthanides making most of the stream, so that this dependence requires a more careful examination.

\section{Influence of the plasma composition}

The influence of plasma composition on the separation capabilities is investigated by modifying the previous numerical model. The trajectory of a single ion (144 amu or $\left.{ }^{241} \mathrm{Am}\right)$ is now computed by considering its interaction with a plasma made of both ion species and electrons. The total plasma density is $n=n^{(144)}+n^{(241)}$, where $n^{(144)}$ and $n^{(241)}$ denote, respectively, the light $(144 \mathrm{amu})$ and heavy $\left({ }^{241} \mathrm{Am}\right)$ ion densities. The plasma composition ratio $\eta=$ $n^{(241)} /\left[n^{(144)}+n^{(241)}\right]$ is introduced in order to investigate the sensitivity of the plasma separation properties as a function of the plasma composition.

Typical results obtained for constant plasma number density (similar to the ones highlighted in figure 3), but varying plasma compositions, are presented in figure 4. Looking at the ${ }^{241} \mathrm{Am}$ data, it can be seen that a decrease in $\eta$, that is to say a decrease in the heavy ion fraction in favor of the lighter ions, leads to an increase in the collection on the heavy side, and a corresponding decrease in the collection on the light side. The separation factor, computed as the ratio of heavy to light streams fraction, varies here from 1.64 for $\eta=1$ to 1.78 for $\eta=0$. A plausible explanation for this evolution is the weaker pitch-angle scattering experienced by heavy ions on light ones, as compared with heavy-heavy ion collisions. However, the consideration of pitch angle scattering is not a sufficient explanation by itself, as demonstrated by the evolution of the data corresponding to light ions (144 amu) in figure 4 . Indeed, although a similar decrease in the pitch angle scattering as $\eta$ decreases is expected to enhance the collection on the heavy side at the expense of the collection to the light 


$$
\begin{aligned}
& n\left[\mathrm{~cm}^{-3}\right] \\
& 6 \times 10^{11} \quad 10^{12} \quad 4 \times 10^{12}
\end{aligned}
$$

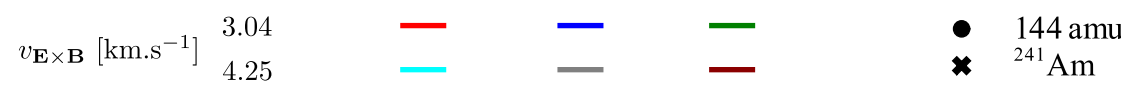

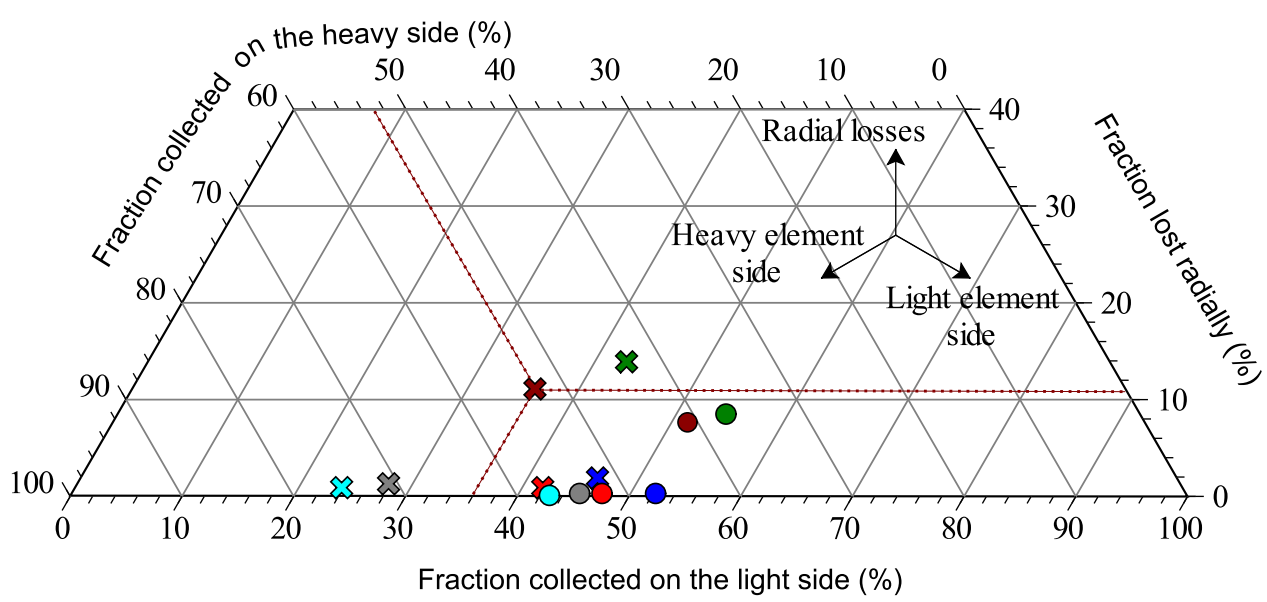

Figure 3. Separation properties obtained for various plasma densities and rotation speeds, and an ion temperature $T_{\mathrm{i}}=20 \mathrm{eV}$. In this figure, the fraction collected on the light side increases from left to right, the fraction collected on the heavy side increases from right to left, and the fraction lost radially increases from bottom to top. As an example, the dark-red cross $\left({ }^{241} \mathrm{Am}, 4 \times 10^{12} \mathrm{~cm}^{-3}, 4.25 \mathrm{~km} \mathrm{~s}^{-1}\right)$ reads $36 \%$ collected on the light side, $53 \%$ collected on the heavy side, and $11 \%$ lost radially.

side, an opposite behavior is observed as $\eta$ decreases under about 0.5 in this particular case.

An explanation for this trend can be proposed by introducing the Langevin friction and diffusion coefficients [20] for a particle $\alpha$ of velocity $v$ interacting with an ion population $\beta$ of isotropic velocity distribution,

$$
\begin{aligned}
& \mathcal{F}^{(\alpha)}(v)=-n^{(\beta)} \Gamma_{p}^{(\alpha \beta)} \frac{8 \pi}{v^{2}} \int_{0}^{v} f^{(\beta)}(w) w^{2} \mathrm{~d} w \\
& \mathcal{D}_{\|}^{(\alpha)}(v)=n^{(\beta)} \Gamma_{p}^{(\alpha \beta)} \frac{8 \pi}{3} \\
& \times\left[\frac{1}{v^{3}} \int_{0}^{v} f^{(\beta)}(w) w^{4} \mathrm{~d} w+\int_{v}^{\infty} f^{(\beta)}(w) w \mathrm{~d} w\right] \\
& \mathcal{D}_{\perp}^{(\alpha)}(v)=n^{(\beta)} \Gamma_{p}^{(\alpha \beta)} \frac{4 \pi}{3}\left[\frac{1}{v^{3}} \int_{0}^{v} f^{(\beta)}(w) w^{2}\left(3 v^{2}-w^{2}\right) \mathrm{d} w\right. \\
& \left.+2 \int_{v}^{\infty} f^{(\beta)}(w) w \mathrm{~d} w\right],
\end{aligned}
$$

where

$$
\Gamma_{p}^{(\alpha \beta)}=q_{\alpha}^{2} q_{\beta}^{2}\left[4 \pi \epsilon_{0}^{2} m_{\alpha}^{2}\right]^{-1 / 2} \ln \Lambda,
$$

with $q$ the particle charge, $m$ the particle mass, $n$ the number density, $\ln \Lambda$ the Coulomb logarithm and $f(v)$ the velocity distribution function. The corresponding coefficients, obtained for a light ion (144 amu) interacting with a plasma composed of two distinct $10 \mathrm{eV}$ Maxwellian distributions $f^{(\beta)}(v)=\left[m^{(\beta)} /\left(2 \pi k_{\mathrm{B}} T_{\mathrm{i}}\right)\right]^{3 / 2} \exp \left[-m^{(\beta)} v^{2} /\left(2 k_{\mathrm{B}} T_{\mathrm{i}}\right)\right]$, where $(\beta)=(144)$ and $(\beta)=(241)$, are plotted in figure 5. As mentioned previously, the perpendicular diffusion coefficient $\mathcal{D}_{\perp}$ increases with $\eta$, meaning that lighter ions are less efficient at redirecting ion velocity vector toward the axial ends of the device than heavier ones. This effect can explain, as invoked for ${ }^{241} \mathrm{Am}$, an increase in the collection on the heavy side at

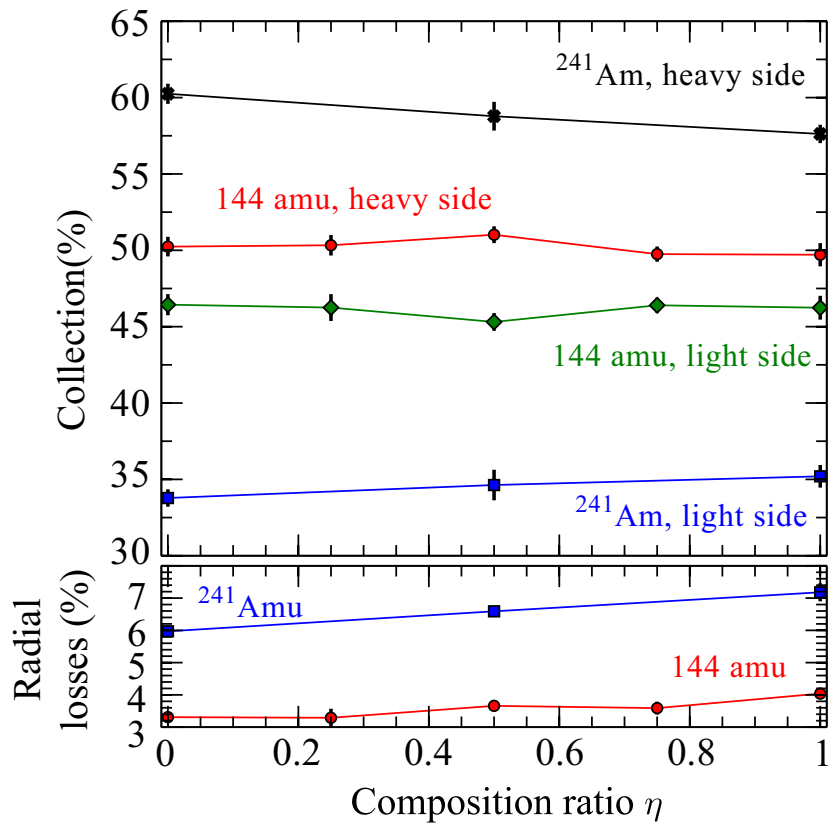

Figure 4. Evolution of the separation properties as a function of the composition ratio $\eta=n^{(241)} /\left[n^{(144)}+n^{(241)}\right]$. Plasma parameters: $n=n^{(144)}+n^{(241)}=10^{12} \mathrm{~cm}^{-3}, T_{\mathrm{i}}=10 \mathrm{eV}$, plasma rotation speed at injection point $v_{E \times B}=E / B \sim 3 \mathrm{~km} \mathrm{~s}^{-1} \sim 0.83 v_{\mathrm{th}}{ }^{(144)}$.

the expense of the light side as $\eta$ decreases. This collisionality decrease also explains the lower radial loss levels observed for low $\eta$ values. However, looking now at the friction coefficient $\mathcal{F}$ in figure $5, \mathcal{F}$ scales as $\eta$ for low $v / v_{\text {th }}$ ratios. This indicates that slowing down decreases as the mean plasma ion mass decreases. Such an effect should limit the collection of ions on the heavy side, since a slower decrease in the ion magnetic 


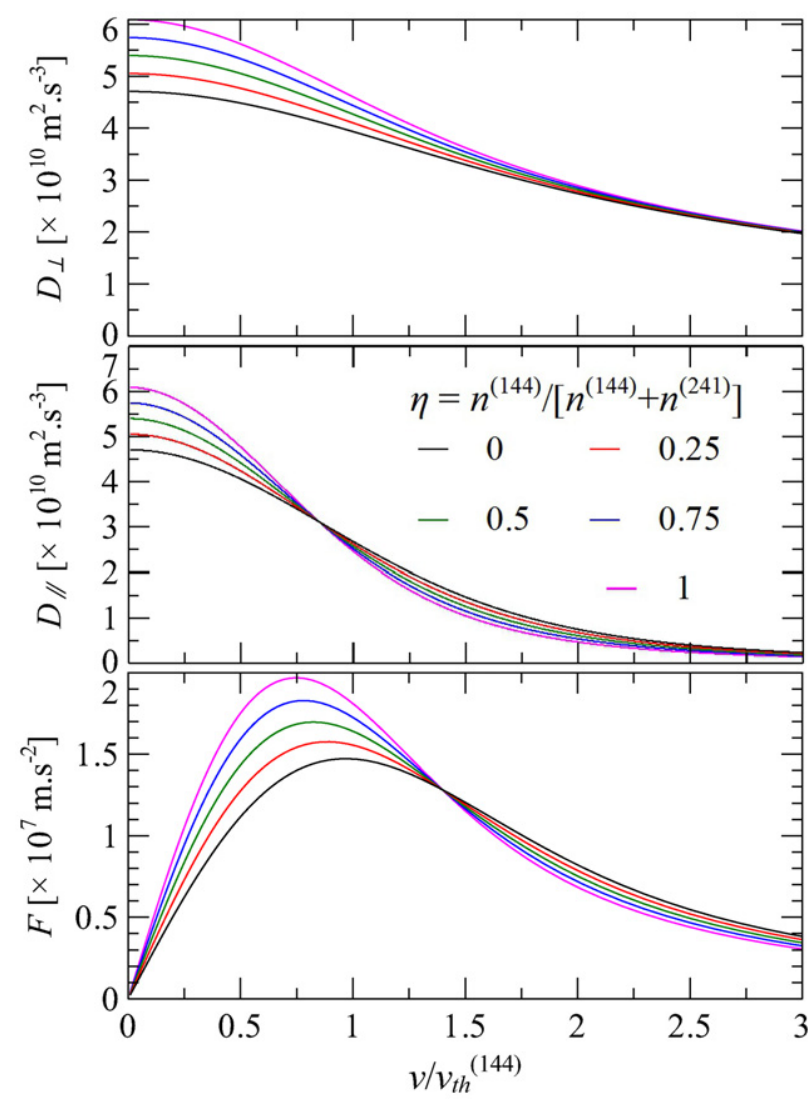

Figure 5. Langevin formalism perpendicular and parallel diffusion coefficients $\left(\mathcal{D}_{\perp}\right.$ and $\left.\mathcal{D}_{\|}\right)$, and friction coefficient $(\mathcal{F})$ for various values of the composition ratio $\eta=n^{(144)} /\left[n^{(144)}+n^{(241)}\right]$. Light ion $\left(m_{\mathrm{L}}=144 \mathrm{amu}\right)$ impacting on a mixture of $144 \mathrm{amu}$ and ${ }^{241} \mathrm{Am}$ ions. Plasma parameters: $n=n^{(144)}+n^{(241)}=10^{12} \mathrm{~cm}^{-3}$, $T_{\mathrm{i}}=10 \mathrm{eV}$ isotropic Maxwellian velocity distribution.

moment $\mu$ makes it harder for the ions to exit through the magnetic mirror. In addition, better confinement on the heavy side naturally brings a larger ion collection on the light side, as the increased confinement time offers better chances for an ion velocity vector to be redirected toward the light collection side. Perpendicular diffusion $\mathcal{D}_{\perp}$ and friction $\mathcal{F}$ dependences on the plasma composition $\eta$ consequently yield opposite effects.

A justification for the difference observed between the light and heavy ion separation property, as it evolves with $\eta$, might lie in the initial velocity of these ions. Ions are initialized at rest in the lab frame, that is to say with the rotation velocity in the rotating frame. In this frame, both ions have consequently the same initial velocity but different kinetic energies $\varepsilon$ as a result of their mass difference. For the $v_{E \times B} \sim 3 \mathrm{~km} \mathrm{~s}^{-1}$ case analyzed, light and heavy ions have initially kinetic energies $\varepsilon^{(144)}=6.9 \mathrm{eV}$ and $\varepsilon^{(241)}=11.7 \mathrm{eV}$. Considering the $T_{\mathrm{i}}=10 \mathrm{eV}$ background ion temperature (i.e. $v_{\text {th }}{ }^{(144)} \sim 3.6 \mathrm{~km} \mathrm{~s}^{-1}$ and $v_{\text {th }}{ }^{(241)} \sim 2.8 \mathrm{~km} \mathrm{~s}^{-1}$ ), heavy ions are initially supra-thermal, while light ions are initially subthermal. Looking at (1), one can show that $\partial \mathcal{F} / \partial \eta$ decreases for $v / v_{\text {th }}{ }^{(144)}>\zeta$, with $\zeta$ defined by

$$
\begin{aligned}
\exp [ & \left.-\zeta^{2}\right]\left(1+\zeta^{2}\right)-\exp \left[-\rho_{m} \zeta^{2}\right]\left(1+\rho_{m} \zeta^{2}\right) \sqrt{\rho_{m}} \\
& =\frac{\sqrt{\pi}}{2 \zeta}\left(\operatorname{Erf}[\zeta]-\operatorname{Erf}\left[\sqrt{\rho_{m}} \zeta\right]\right),
\end{aligned}
$$

where $\rho_{m}=m^{(241)} / m^{(144)}$, and $\operatorname{Erf}[x]=\int_{0}^{x} \exp \left(-t^{2}\right) \mathrm{d} t$ is the error function. Solving (5) for the ion masses and plasma ion temperature considered, one gets $\zeta \sim 0.61$. The influence of a plasma mean mass decrease on $\mathcal{F}$ is consequently expected to be larger for smaller $v / v_{\text {th }}$ ratios, such as likely to occur for light elements. This could explain the fact that the separation increase with decreasing $\eta$ is partly mitigated for light ions, but not for heavy ions.

In summary, it appears that the lower the fraction of heavy elements in the input stream, the higher the separation factor for heavy elements when the background ion temperature is set in between the light and heavy initial rotation energies. A low heavy element fraction is typical of the separation of americium from lanthanides (see the appendix). Effects on the light elements separation are observed to be more complex and less distinct. A proposed explanation for this difference is the larger influence of the slowing down dependence on the plasma mass composition of light elements.

\section{Summary}

Because plasma consists of dissociated ions and electrons, rather than chemical compounds, it offers unique capabilities for separating elements as compared with liquid or gaseous states. This is, for example, the case for the separation of elements exhibiting strong chemical similarities, for which chemical techniques appear especially challenging. Such a separation need is, for example, encountered in the context of nuclear spent fuel reprocessing, in the form of the removal of ${ }^{241} \mathrm{Am}$ from a mixture of lanthanide elements.

By turning the material to be separated into a plasma, one can then take advantage of asymmetric plasma confinement properties to separate elements. In the case of the magnetic centrifugal mass filter, the confinement asymmetry is provided by means of the centrifugal and magnetic forces exerted on the charged particles composing the plasma. Numerical simulations indicate that this particular plasma filter configuration might offer separation factors of up to three in a single pass for the particular case of removing ${ }^{241} \mathrm{Am}$ from lanthanides, and that for plasma number densities of about $10^{12} \mathrm{~cm}^{-3}$. Parameter space probing shows that the separation properties can be tuned on a wide range by varying the rotation speed and/or the plasma number densities, while maintaining radial losses to acceptable levels $(<15 \%)$. In addition, it is expected that increasing the device dimensions would limit the radial losses.

Such densities, combined with ion temperatures of the order of $10 \mathrm{eV}$, would yield a throughput of about $10 \mathrm{MT}$ (metric ton) per year for a $0.4 \mathrm{~m}$ radius device. Largest chemical reprocessing plants' capabilities (e.g. La Hague, France) are of the order of $1000 \mathrm{MT}$ of spent fuel per year, that is to say of the order of $1 \mathrm{MT}$ of actinides and $10 \mathrm{MT}$ of lanthanides per year. A single plasma unit could hence in principle ensure the back-end separation of actinides from lanthanides. One should, however, expect some level of sequential processing or staging to be required in order to meet the separation requirements. A $99 \%$ purification would, for 


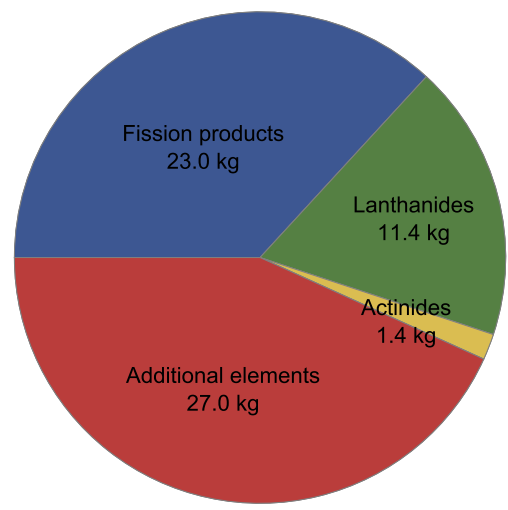

(a) Oxides content by element group, per metric ton of Uranium

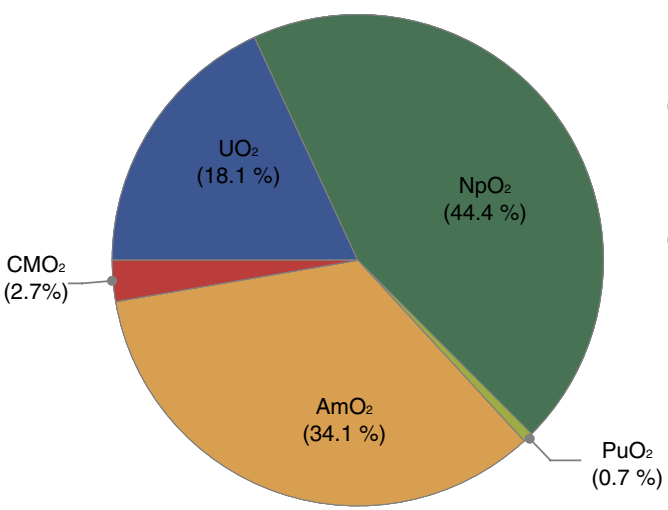

(b) Distribution within actinides elements

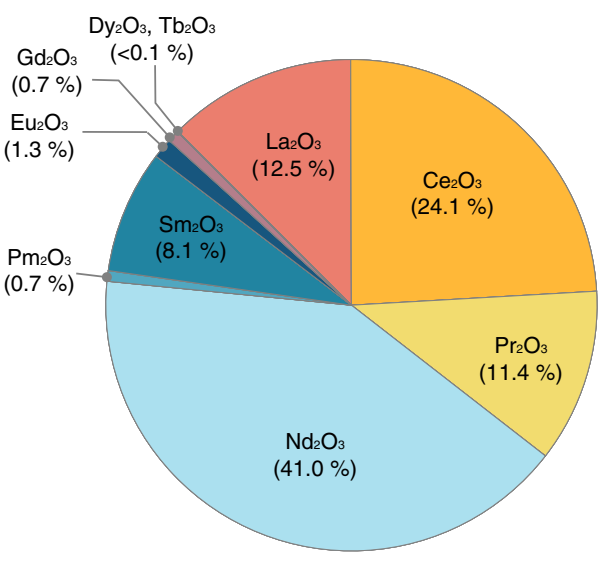

(c) Distribution within lanthanides elements

Figure A1. Composition of nuclear spent fuel for a standard $33 \mathrm{GW} / \mathrm{t}$ reactor, after 3 year cooling, data from [22].

example, require about eight passes, or alternatively a cascade of eight units, for a symmetric plasma composition.

For asymmetric plasma compositions where most of the mass is made of light ions, the heavy species tend to separate better than the light species. This is foreseen as an advantage for the particular case of ${ }^{241} \mathrm{Am}$-lanthanide separation since the mass of ${ }^{241} \mathrm{Am}$ is evaluated to be about only $4.2 \%$ of the input stream. More generally, this property would be particularly interesting for any separation process of which the objective consists mainly in isolating heavy elements, such as nuclear waste remediation.

\section{Acknowledgment}

This work was supported by US DOE under contract DEAC02-09CH11466.

\section{Appendix. Optimized nuclear spent fuel cycle requirements}

Nuclear spent fuel recovered from reactors consists mostly (about 94\% in mass) of unburned uranium fuel. This unaffected fuel can be separated and processed for additional use, through the Purex (plutonium uranium refining by extraction) process [21]. This process, globally in use around the world in reprocessing plants, allows recovering about $99.9 \%$ of both the uranium and plutonium present in the spent fuel stream. As shown in figure A1, the rest of the stream (over 40 different elements) is made of various fission products, lanthanides, minor actinides, as well as additional elements resulting from the PUREX treatment. The current industrial approach consists in stocking this stream for a year before vitrification and permanent geological repository.

At this stage, the large majority of the long-term radioactivity hazard originates from the minor actinides and daughter elements. Looking at figure $\mathrm{A} 1(b)$, one can see that, apart from the non-recovered uranium and plutonium, the hazard lies in the presence of neptunium, americium and curium. Transmutation of these elements into shorter-lived elements is one of the options for decreasing the spent fuel long-term biological hazard. However, because of the large neutron capture cross-section exhibited by lanthanides [14], actinides have to be separated from lanthanides a priori. The usually accepted goal for this partitioning step is a separation efficiency of $99.9 \%$. An upgrade of the chemical PUREX process, called the Advanced PUREX, has been developed to recover neptunium in addition to uranium and plutonium [23]. On the other hand, the chemical separation of americium and curium from the lanthanides is made particularly difficult because of the chemical similarities existing between these elements $[11,15]$. Note that among these two elements, only 


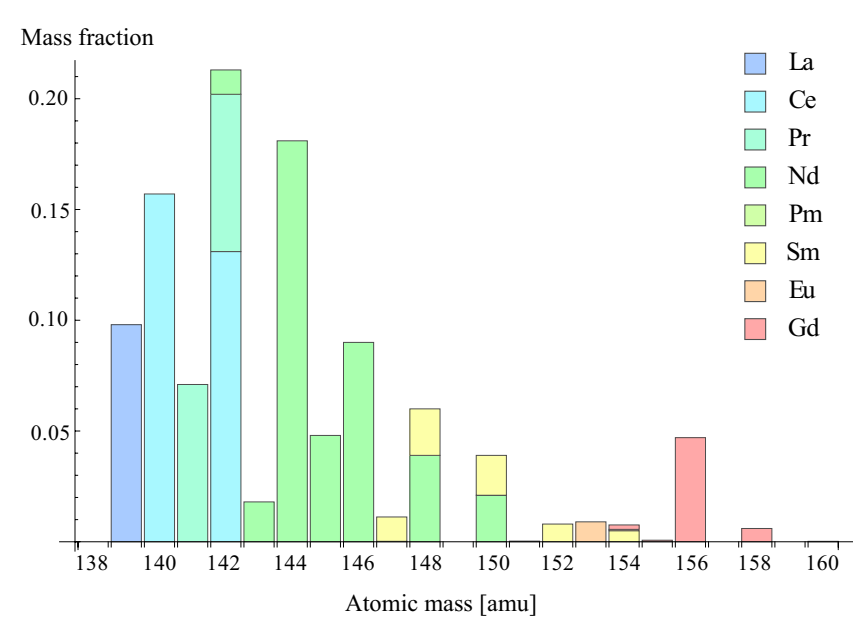

Figure A2. Isotopic distribution of mass within the lanthanide fission products, from [24]. The average mass is $144 \mathrm{amu}$.

the removal of americium is strictly required. As a matter of fact, the two main curium isotopes $\left({ }^{243} \mathrm{Cm}\right.$ and $\left.{ }^{244} \mathrm{Cm}\right)$ decay into plutonium $\left({ }^{239} \mathrm{Pu}\right.$ and $\left.{ }^{240} \mathrm{Pu}\right)$, with half-lives of, respectively, 29 and 18 years. Curium could therefore be stored and monitored with the lanthanides, until the naturally formed plutonium can be recovered. On the other hand, ${ }^{241} \mathrm{Am}$, which is the main americium isotope, decays into neptunium with a half-life of about 432 years, making its a priori removal a requirement.

The interest of a separation scheme based on element mass is illustrated by the lanthanide group element distribution, as depicted in figure $\mathrm{A} 1(c)$. More precisely, the isotopic mass distribution within the lanthanide group [24], as plotted in figure A2, indicates an average mass of $144 \mathrm{amu}$. The large difference as compared with americium's mass makes a mass based separation scheme practical and attractive.

\section{References}

[1] Lawrence E O 1958 Calutron system US Patent \#2847576, August 1958

[2] Yergey A L and Yergey A 1997 J. Am. Soc. Mass Spectrom. $8943-53$
[3] Lehnert B 1971 Nucl. Fusion 11485

[4] Krishnan M, Geva M and Hirshfield J L 1981 Phys. Rev. Lett. 46 36-8

[5] Grossman M W and Shepp T A 1991 IEEE Trans. Plasma Sci. 19 1114-22

[6] Rax J M, Robiche J and Fisch N J 2007 Phys. Plasmas 14 043102-8

[7] Freeman R et al 2003 AIP Conf. Proc. 694 403-10

[8] Ohkawa T and Miller R L 2002 Phys. Plasmas 9 5116-20

[9] Fetterman A J and Fisch N J 2011 Phys. Plasmas 18094503

[10] Gueroult R and Fisch N J 2012 Phys. Plasmas 19122503

[11] Peterman D R, Greenhalgh M R, Tillotson R D, Klaehn J R, Harrup M K, Luther T A, Law J D and Daniels L M 2008 Separation of minor actinides from lanthanides by dithiophosphinic acid extractants Proc. 2008 Int. Solvent Extraction Conf. (ISEC)

[12] Magill J, Berthou V, Haas D, Galy J, Schenkel R, Wiese H W, Heusener G, Tommasi J and Youinou G 2003 Nucl. Energy 42 263-77

[13] Blue Ribbon Commission on America's Nuclear Future 2012 Reactor and Fuel Cycle Technology Subcommittee Report to the Full Commission Updated Report (Washington, DC: Blue Ribbon Commission on America's Nuclear Future)

[14] Mathur J N, Murali M S and Nash K L 2001 Solvent Extraction and Ion Exchange 19 357-90

[15] Morss L R, Edelstein N M and Fuger J (ed) 2011 The Chemistry of the Actinide and Transactinide Elements (Berlin: Springer)

[16] Boswell R 1970 Phys. Lett. A 33 457-8

[17] Tanaka Y, Pigarov A Y, Smirnov R D, Krasheninnikov S I, Ohno N and Uesugi Y 2007 Phys. Plasmas 14052504

[18] Post D, Jensen R, Tarter C, Grasberger W and Lokke W 1977 At. Data Nucl. Data Tables 20 397-439

[19] Gueroult R, Rax J M and Fisch N J 2014 Phys. Plasmas 21020701

[20] Manheimer W M, Lampe M and Joyce G 1997 J. Comput. Phys. 138 563-84

[21] Anderson H H 1960 Solvent extraction process for plutonium US Patent \#2924506, February 1960

[22] Bardez I 2004 Etude des caractéristiques structurales et des propriétés de verres riches en terres rares destinés au confinement des produits de fission et éléments à vie longue PhD Thesis Université Pierre et Marie Curie

[23] Taylor R J, Gregson C R, Carrott M J, Mason C and Sarsfield M J 2013 Solvent Extraction Ion Exch. 31 442-62

[24] Alonso J I G, Sena F, Arbore P, Betti M and Koch L 1995 J. Anal. At. Spectrom. 10 381-93 